\title{
Identification of a Carboxy-Terminal Glutamine-Rich Domain in Agrobacterium tumefaciens Coupling Protein VirD4 Required for Recognition of T-Strand DNA and Not VirE2 as a Substrate for Transfer to Plant Cells
}

\author{
Anath Das ${ }^{\dagger}$ \\ Department of Biochemistry, Molecular Biology and Biophysics, and Microbial and Plant Genomics Institute, University of \\ Minnesota, Minneapolis, MN 55455, U.S.A.
}

Accepted 10 October 2019.

Agrobacterium tumefaciens transfers DNA and proteins to a plant cell inciting crown gall tumor disease on most plants. VirD4 targets the DNA and protein substrates to a type IV secretion (T4S) apparatus for translocation into the plant cell. Several bacteria with VirD4 homologs use T4S for intercellular export of microbial macromolecules to eukaryotic and prokaryotic hosts. How the VirD4 proteins recognize the diverse substrates is not well understood. To identify functional domains of $A$. tumefaciens pTiA6 VirD4, we introduced random 19-codon and targeted 10-codon insertions throughout the coding region. Analysis of 21 mutants showed that only the carboxy-terminal end of VirD4 is tolerant of an insertion. Sequence comparison of VirD4 proteins of Agrobacterium spp. and their close relative, Rhizobium etli, showed that these proteins contain a highly conserved $C$-terminal end, but the immediate upstream regions share no discernible sequence similarity. The conserved region sequence is rich in the amino acid glutamine (6/13 Q). Using site-specific and deletion mutagenesis, we demonstrated that the conserved Q-rich region is required for VirD4 function and for the specific recognition of VirD2-linked T-strand DNA as a substrate for translocation to plants. The Q-rich region is not required for the transfer of a second $A$. tumefaciens substrate, VirE2, to plants or a promiscuous Escherichia coli IncQ plasmid to another A. tumefaciens strain. We identified Q-rich sequences at or near the $\mathrm{C}$ terminus of several VirD4 homologs, including the $E$. coli $\mathrm{F}$ plasmid TraD. In F TraD, the Q-rich sequence maps to a region required specifically for the conjugative transfer of the $F$ plasmid.

Agrobacterium tumefaciens causes crown gall tumor disease on dicotyledonous plants by transferring oncogenic DNA to plant cells using a type IV secretion (T4S) apparatus (Chilton

${ }^{\dagger}$ Corresponding author: A. Das; dasxx002@umn.edu

Funding: This work was supported by a grant from the Agricultural Experiment Station, University of Minnesota.

*The $\boldsymbol{e}$-Xtra logo stands for "electronic extra" and indicates that three supplementary figures and one supplementary table are published online.

The author(s) declare there is no conflict of interest.

(c) 2020 The American Phytopathological Society et al. 1977; Vergunst et al. 2000). T4S is used by bacteria to transfer large macromolecules across cell membranes into other bacteria and eukaryotes (Christie 2001). Rapid evolution of multidrug-resistant bacteria can be a result of efficient horizontal transfer of plasmid DNA among bacteria using the T4S system. Human, animal, and plant pathogens use T4S to cause diseases on eukaryotic hosts. Substrate translocation during Agrobacterium-mediated substrate transfer requires the participation of 12 virulence proteins, VirB1 to VirB11 and VirD4 (Berger and Christie 1994; Vergunst et al. 2000). Many of these proteins are conserved in bacteria harboring a T4S system (Christie 2001; Christie et al. 2014). The VirB proteins assemble a multiprotein translocation apparatus spanning both bacterial membranes, while the coupling protein VirD4 functions by targeting a substrate to the apparatus for delivery into a recipient cell (Cabezón et al. 1997; Fronzes et al. 2009). The number of substrates translocated to a host varies widely, ranging from a few in Agrobacterium and Helicobacter species to hundreds in Legionella species (Christie et al. 2014; Escoll et al. 2013). Molecular mechanisms of substrate recognition by the VirD4 family of coupling proteins are not well understood.

For DNA transfer to plants, the A. tumefaciens Ti-plasmid is processed at the T-DNA border sequences, delineated by a conserved 24-bp direct repeat sequence at the two termini, by the VirD1 and VirD2 relaxase, producing a singlestranded T-strand DNA molecule covalently attached to VirD2 (Christie 2001). VirD4 escorts the VirD2-T-strand DNA intermediate to the T4S apparatus for secretion (Cascales et al. 2005). A few proteins, including VirD5, VirE2, VirE3, and VirF, as well as A. rhizogenes GALLS-FL and GALLS-CT, also are exported to a plant cell in a VirD4dependent manner (Gelvin 2012). VirE2 is a single-stranded DNA-binding protein whose protective function against nucleases is required in the plant cell (Das 1988; Simone et al. 2001).

VirD4 is an integral membrane protein with two transmembrane (TM) domains near the $\mathrm{N}$ terminus (Das and Xie 1998). The small periplasmic domain is required for DNA transfer and for the localization of VirD4 to a cell pole (Kumar and Das 2002). This domain also functions in the handover of a DNA substrate from VirD4 to VirB11 and other VirB channel proteins in the transfer pathway (Cascales et al. 2013). Immediately after the second TM domain, VirD4 contains ATPbinding Walker $\mathrm{A}$ and $\mathrm{B}$ motifs that are essential for DNA transfer (Cascales et al. 2013; Kumar and Das 2002). The soluble 
domain of a VirD4 homolog, the Escherichia coli plasmid R388 TrwB $\Delta N 70$, binds ATP, which is required for the conjugative transfer of the plasmid (Tato et al. 2007). These studies suggest a role for ATP utilization in VirD4 function.

TrwB $\Delta N 70$ is a homo-hexameric protein with a central opening of $20 \AA$ capable of accommodating large substrates (Gomis-Rüth et al. 2001 and 2002). A helical bundle, known as the all-alpha domain (AAD), sits at the entrance of the channel. Biochemical studies with $A$. tumefaciens VirD4 suggest that the $\mathrm{AAD}$ region participates in the recognition of DNA, and possibly proteins, as substrates (Whitaker et al. 2015). Members of the VirD4 protein family share significant sequence similarity throughout most of the protein, except for the extreme $\mathrm{C}$ terminus. The $\mathrm{C}$ terminus of the pTiA6 VirD4, however, is required for function (Kumar and Das 2002; Whitaker et al. 2016). This region in the VirD4 family of proteins is highly variable, both in primary sequence and in its length. These differences have led to the postulate that the region participates in recognition of system-specific substrates. A recent study using chimeric VirD4 proteins supports this hypothesis (Whitaker et al. 2016).

The introduction of short (10 to 30 amino acids) random sequences within a protein could adversely affect its function (Nelson et al. 1997). This property has been used successfully to identify protein functional domains and to introduce detection tags (Haft et al. 2007; Waters and Dunny 2001). To identify functional domains of pTiA6 VirD4, we introduced 19- and 10-codon insertions by transposon and site-specific mutagenesis, respectively. We demonstrate that only the Cterminal end of VirD4 is tolerant of an insertion. Hidden within this region we identified a conserved 13-residue glutamine-rich domain $(46 \% \mathrm{Q})$ that is required for DNA transfer to plants. This domain is essential for the recognition of the T-strand DNA linked to VirD2 as a substrate for transfer to plants. We also show that the Q-rich domain is not required for the transfer of a second A. tumefaciens substrate, VirE2, to plant cells or of the promiscuous IncQ plasmid pKT230 DNA to another A. tumefaciens strain. Our results suggest that, at a minimum, two separate VirD4 domains are involved in the recognition of substrates targeted for translocation via a T4S system.

\section{RESULTS}

\section{Insertional mutagenesis of virD4.}

To probe for functional domains of $A$. tumefaciens pTiA6 VirD4, we introduced random insertions of 19 codons within the coding region of a cloned $v i r D_{\mathrm{p}}$-virD4 gene by EZ-tn5 transposon mutagenesis. Analysis of plasmid DNA from 120 kanamycin-resistant colonies by restriction mapping and DNA sequence analysis led to the identification of 11 unique insertions in the virD4 coding region. The insertions mapped throughout the gene; however, a few regions were devoid of an insertion (Fig. 1). To fill in the gaps, 10 additional mutants with 10-codon insertions at targeted sites were generated by site-specific mutagenesis (Kumar and Das 2002). Analysis of 21 mutants covering the virD4 coding region showed that an insertion is not tolerated in most of the protein except for the $\mathrm{C}$ terminus (Fig. 1). Sixteen insertions that mapped within the first 542 residues produced proteins that were nonfunctional in T-DNA transfer in complementation assays. In contrast, four insertions mapping within the $\mathrm{C}$-terminal 103 residues, at codons 554, 580, 609, and 655 (the penultimate codon), had no effect on protein function. A fifth insertion mapping to this region, at codon 640 (VirD4 ${ }_{i 640}$ ), did render the protein nonfunctional, indicating that the extreme $\mathrm{C}$ terminus is required for T-DNA transfer. All mutant proteins, except for two $\left(\operatorname{VirD}_{\mathrm{i} 291}\right.$ and $\left.\operatorname{VirD}_{\mathrm{i} 332}\right)$, were stably expressed in the cell (Supplementary Fig. S1).

\section{A virD4 mutant proficient in VirE2 transfer but deficient in T-strand DNA transfer.}

T-DNA and VirE2 protein are independently transferred into a plant cell by A. tumefaciens (Binns et al. 1995). We studied the effect of an insertion in virD4 on T-DNA transfer and VirE2 transfer using in-planta complementation assays (also known as extracellular complementation tests [Otten et al. 1984]). All avirulent mutants, except for one, were deficient in the independent transfer of the two substrates (Fig. 1). The exception was VirD $4_{i 640}$, a mutant deficient in T-DNA transfer but proficient in VirE2 transfer (Fig. 1). The novel phenotype of VirD4 ${ }_{i 640}$ suggests that the $\mathrm{C}$ terminus of VirD4 encodes a VirD2-linked Tstrand DNA recognition motif and that this motif is not

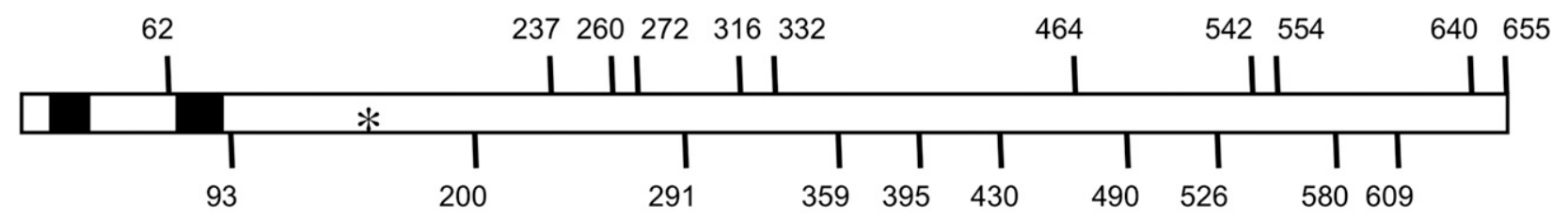

\section{$\Delta$ virD4 comple- mentation}

\section{T-strand transfer}

\section{VirE2 transfer}

Fig. 1. Insertional mutagenesis of virD4. An insertion at virD4 codon " $n$ " is indicated by a vertical line. The two transmembrane domains (dark boxes) and the Walker A motif (*) are also shown. Lines above the box identify insertions obtained by EZ-tn5 mutagenesis, and lines below identify those introduced by sitespecific mutagenesis. The effect of a mutation on VirD4 function was monitored by complementation of the virD4 deletion mutant Agrobacterium tumefaciens A348 D 4. The effect on either T-strand DNA or VirE2 transfer to plants was monitored by in planta complementation assays by coinoculation with $A$. tumefaciens MX358 (virE2 ${ }^{-}$) or PC2760 (T-DNA ${ }^{-}$). -: avirulent; +: virulent. Insertions at codons 554, 580, 609, and 655 are proficient in both T-DNA and VirE2 transfer based on their virulent phenotype in virD4 complementation assays. 
required for the transfer of the second substrate, VirE2. The recognition motifs for the two substrates, therefore, are likely to be different.

\section{A conserved glutamine-rich region at the $\mathbf{C}$ terminus of Agrobacterium sp. VirD4 proteins.}

The unique phenotype of VirD $4_{i 640}$ prompted us to take a closer look at the primary sequences of the C-terminal end of this family of proteins. All VirD4 family members share significant sequence similarity throughout the protein except in the C-terminal region, where similarity is virtually nonexistent (Supplementary Fig. S2). Diversity in the C-terminal sequences could provide the flexibility necessary for the recognition of system-specific substrates exported by the VirD4 homologs. This region, thus, has been postulated to function in substrate recognition, and a recent study supports this hypothesis (Whitaker et al. 2016). Substrates transferred by the pathogenic Agrobacterium sp., however, are similar, and therefore, the conservation of a substrate recognition motif is expected in this subfamily. To test this hypothesis, we compared the primary sequences of the C-terminal 100 residues (the only region with several silent insertions [Fig. 1]) of VirD4 proteins of Agrobacterium sp. and a close relative, Rhizobium etli. Surprisingly, the proximal (N-terminal) approximately 70 residues showed no discernible sequence similarity, but the C-terminal approximately 30-amino acid region was highly conserved (Fig. 2; Supplementary Fig. S3). These findings, in conjunction with the phenotype of the mutants discussed previously, led us to postulate that a substrate recognition site resides at the extreme $\mathrm{C}$ terminus of VirD4 proteins and that the immediately upstream region serves as a spacer.

A unique feature of the C-terminal conserved region (residues 624 to 646 in pTiA6 VirD4) is that it contains a small segment rich in the amino acid glutamine (Fig. 2). Within a 13amino acid region, residues 633 to 645 , six are glutamine residues (hereafter referred to as the Q-rich domain). Interspersed among the glutamine residues are five invariant leucine and isoleucine residues. Two invariant alanine and arginine residues border the $\mathrm{N}$ - and $\mathrm{C}$-terminal sides, respectively, and several additional conserved residues are present on the N-terminal side of the Q-rich domain.
Functional analysis of the Q-rich domain.

Mutational studies were performed to determine the role of the Q-rich domain in T-DNA transfer. We introduced amino acid substitutions and small deletions within the Q-rich domain and studied the effect of a mutation on VirD4 function by complementation assays. Alanine substitutions of the invariant Q633 (Q633A) and of both invariant Q644 and near invariant Q645 (QQ64445AA) abolished VirD4 function (Fig. 3A and B). A triple mutant with glutamine to asparagine substitution of residues 633 to 635 (QQQ633-35NNN) exhibited near avirulent phenotype. This mutant formed a few small tumors only after prolonged infection (10 to 12 weeks postinfection). Two double mutants with alanine substitutions of the two invariant leucine residues at position 636 and 637 (LL63637AA) and the two invariant isoleucine residues at 640 and 641 (II64041AA) also were nonfunctional. A substitution of aspartic acid 638 to valine (D638V), on the other hand, had no effect on VirD4 function. The conserved residues within the Q-rich domain, therefore, are required for VirD4 function.

To map the domain more precisely, we analyzed five deletion mutants (Fig. 3A and B). Deletion of six codons (633 to 638) within the first half of the Q-rich domain rendered the protein nonfunctional. Deletion of three amino acids at the other end, codons 646 to 648, had no effect on VirD4 function. Two deletions in the extreme $\mathrm{C}$ terminus were introduced by deleting codons 631 to 656 and by changing codon 648 to a stop codon (resulting in the deletion of codons 648 to 656; R648X). In complementation assays, VirD4 $\Delta 631-656$ had no DNA transfer activity, while VirD4R648X was fully functional. The last nine nonconserved residues of VirD4 are not required, therefore, for protein function and are not part of the Q-rich domain.

Sequences upstream of the Q-rich sequences are not conserved among the Ti- and Ri-plasmid VirD4s (Supplementary Fig. S3). To investigate the role of these sequences in VirD4 function, we introduced a large deletion of codons 561 to 620 . The mutant protein, VirD4 $\Delta 561-620$, was fully functional in tumor formation (Fig. 3B). T-DNA transfer thus requires VirD4 residues 631 to 646, which include a conserved Q-rich domain, and its immediately upstream region has no role in VirD4 function.

\begin{abstract}
pTiA6
pTiBo5 42

pTiC58

pTi-Sakura

A. vitis

pRiA4b

pRi1724

R. etli

consensus

621 VVPPELTLALAAQQQLLDQIIALQQRSRSAPAQPAK 621 VVPPELTLALTAQQQLLDQIIALQQRSRSAPA---630 GVSAEMAPAMIAQQQLLEQIIALQQRYGPASSHSVK 633 GVSAEMAPAMIAQQQLLEQIIALQQRYGPASSHSVK 631 EELLDIAPALLAQRELLEQI ISLQQRGGTDSGT--636 SVPAEMAPALSAQQQLLGQI IALQQRYRPVSSNPIE 628 EKLPEIAPALLAQRELLDQIISLQLRGRTASGT--631 GPSAEVAPALVAQEQLLKRIIALQQRYRSGSSNPGD

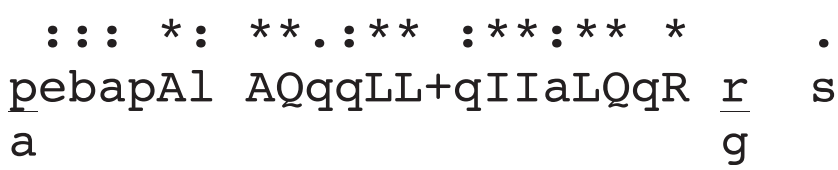

Fig. 2. Sequence conservation near the $\mathrm{C}$ terminus of Agrobacterium and Rhizobium spp. VirD4 proteins. Sequence similarity near the C terminus of the closely related VirD4 proteins is shown. A consensus sequence with conserved residues in capital letters and near conserved residues ( $\geq 6 / 8)$ in small letters is also shown. *: invariant; +: acidic; b: bulky. No sequence conservation was observed within the preceding 70 amino acids. pTiA6 (accession number NP_059816.1), pTiBo542 (AAZ50535), pTiC58 (AAA91606.1), pTi-Sakura (NP_053398.1), A. vitis S4 (ACM39656.1), pRiA4b (P13464.1), pRi1724 (NP_066751.1), and R. etli CFN 42 (YP_471662.1).
\end{abstract}


We used in planta complementation assays to investigate whether the inability of a mutant protein to complement the $A$. tumefaciens deletion strain A348DD4 was due to a defect in the transfer of T-strand DNA, VirE2, or both substrates. All complementation-deficient mutants failed to transfer T-strand DNA but were fully competent in VirE2 transfer (Fig. 3A and C). The VirE2 transfer-proficient phenotype of these mutants and that of VirD4 $\Delta 631-656$, a mutant with a complete deletion in the Q-rich domain, indicates that different VirD4 domains participate in recognition of the two substrates. The Q-rich domain is required for the recognition of the VirD2 linked Tstrand DNA substrate but not VirE2. The VirE2 recognition domain, therefore, must lie elsewhere in the protein.

Effect of mutations in virD4 on conjugative transfer of IncQ plasmid to another A. tumefaciens strain.

The A. tumefaciens T4S system supports conjugative transfer of promiscuous IncQ plasmids into another bacterium (Beijersbergen et al. 1992). We studied the effect of the mutations in virD4 on conjugative transfer of plasmid pKT230 to another A. tumefaciens strain. All insertion mutants that were defective in T-DNA transfer to plants, except for virD $4_{\mathrm{i} 640}$, were also defective in pKT230 transfer to the recipient bacterium (Fig. 4). virD4 $4_{\mathrm{i} 640}$, which is deficient in T-DNA transfer, was competent in pKT230 DNA transfer. All mutants mapping to the Q-rich domain also were proficient in plasmid DNA transfer. One mutant, virD4Q633A, was less efficient in pKT230 DNA transfer. The Q-rich domain and the C-terminal end of VirD4, therefore, are not required for the transfer of IncQ plasmid DNA to another bacterium, and a second DNA recognition domain must be present within the first 554 residues of the protein.

\section{DISCUSSION}

The VirD4 coupling proteins target DNA and protein substrates to the T4S apparatus for transfer to a recipient cell.

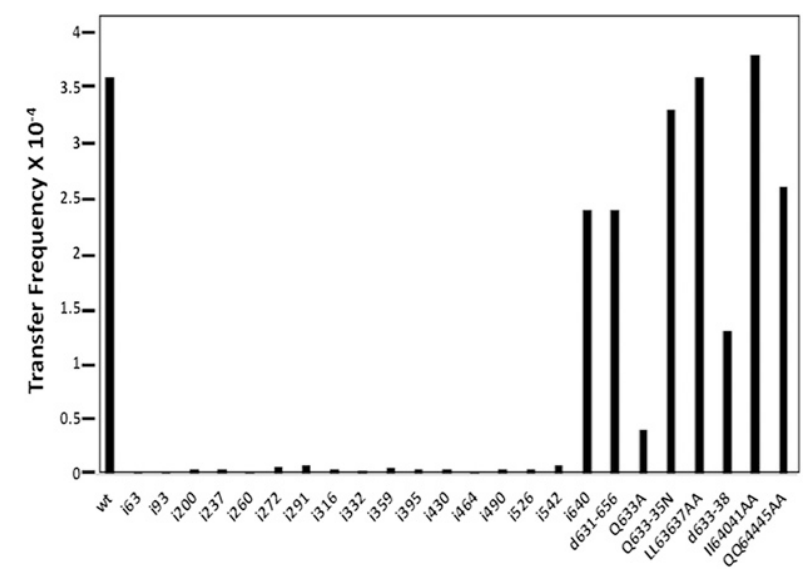

Fig. 4. Effect of a mutation in virD4 in conjugative transfer of IncQ plasmid pKT230 is shown. Transfer of plasmid pKT230 from a virD4 mutant strain to another A. tumefaciens. strain was monitored. $\mathrm{VirD} 4_{1640}$ and all Q-rich domain mutants were proficient in DNA transfer. VirD4Q633A exhibited a low efficiency of transfer. Data presented are an average of two independent experiments.

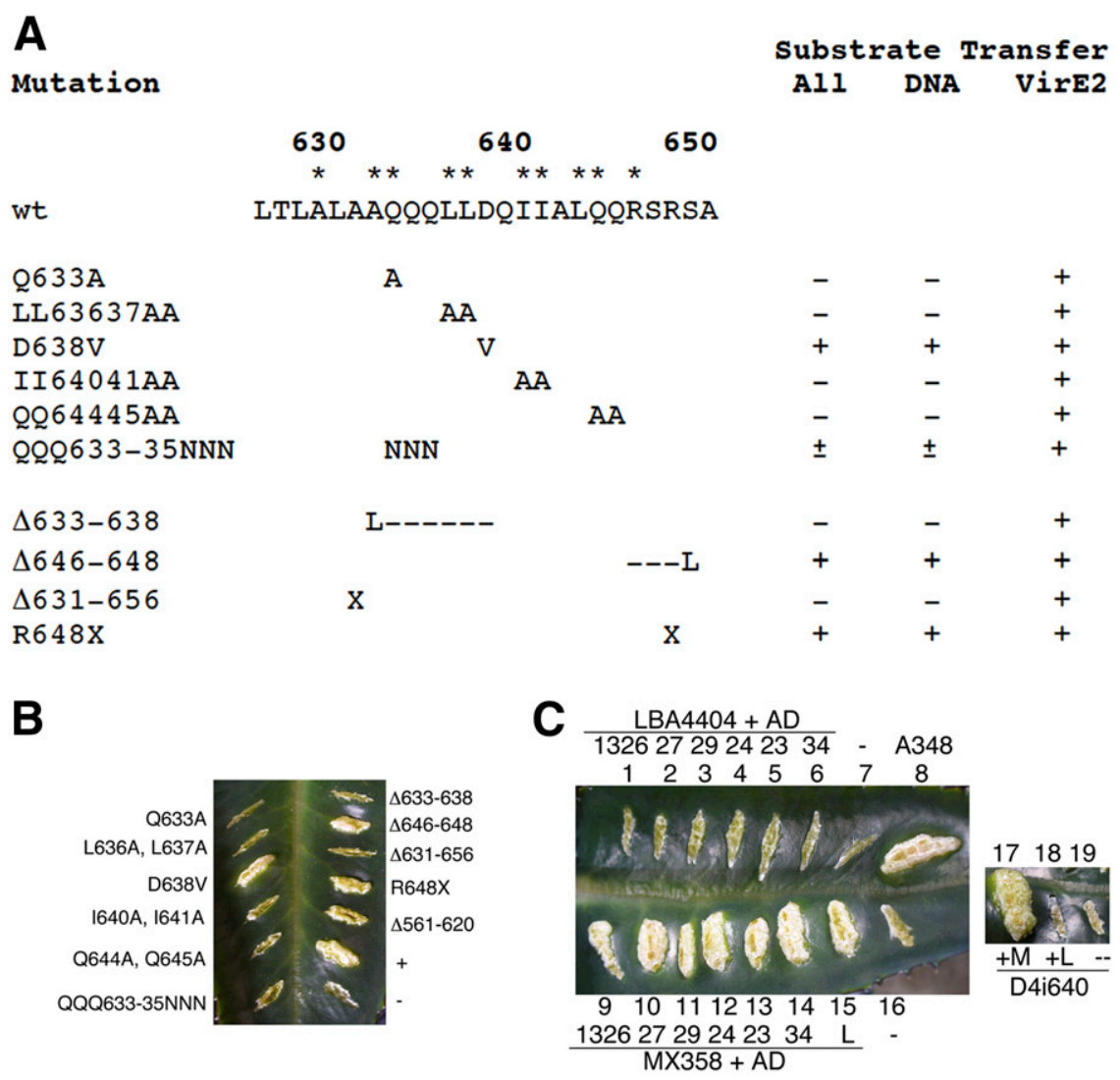

Fig. 3. Role of the VirD4 C-terminal Q-rich region in substrate recognition. A, Effect of amino acid substitutions and deletions within the Q-rich domain on VirD4 function was analyzed by complementation assays. *: Invariant residues; -: deletion; x: stop codon; +: proficient; -: no transfer; \pm : very weakly proficient. B, Phenotype of mutants in virD4 complementation assays is shown. +: A348; -: A136. C, Ability of a virD4 mutant to transfer T-DNA (+LBA4404 [L]) or VirE2 (+MX358 [M]) by in planta complementation assays is shown. AD1326 (virD4Q633A), 1327 (LL63637AA), 1329 (II64041AA), 1324

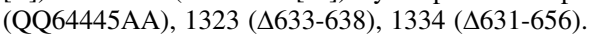


Mechanisms of substrate recognition and secretion are largely unknown. Protein-protein interaction is expected to play a primary role in substrate recognition. Functional analysis of A. tumefaciens virD4 by insertional mutagenesis showed that more than three-fourths of the $\mathrm{N}$-terminal region of the protein could not be modified without a loss of the DNA transfer function (Fig. 1). Several mutants we analyzed had an insertion of 10 amino acids, a short insertion commonly tolerated by proteins. The insertions mapped primarily to four regions: the periplasmic domain (approximately aa 31 to 67 ) ( $\operatorname{VirD}_{i 62}$ ); the nucleotide-binding Walker B motif (VirD4 $\left.{ }_{i 395}\right)$; the AAD domain (approximately aa 201 to 346, according to [Whitaker et al. 2015]) $\left(\operatorname{VirD}_{\mathrm{i} 200}, \mathrm{VirD}_{\mathrm{i} 237}, \mathrm{VirD}_{\mathrm{i} 260}, \operatorname{VirD}_{\mathrm{i} 272}\right.$, and VirD4 ${ }_{i 316}$ ); and the inner channel (VirD4 $4_{i 430}$ and $\operatorname{VirD}_{\mathrm{i} 464}$ ), membrane-proximal channel end (Gomis-Rüth et al. 2001) $\left(\mathrm{VirD}_{\mathrm{i} 93}\right.$ and $\mathrm{VirD}_{\mathrm{i} 490}$ ) and C-terminal end (VirD4 ${ }_{\mathrm{i} 526}$, $\operatorname{VirD}_{\mathrm{i} 542}$, VirD4 $_{\mathrm{i} 554}$, VirD4 $_{\mathrm{i} 580}$, VirD4 $_{\mathrm{i} 609}$, VirD4 $_{\mathrm{i} 640}$, and VirD $_{i 655}$ ). Only four insertions $\left(V_{i r D} 4_{i 554}, \operatorname{VirD}_{i 580}\right.$, VirD4 ${ }_{i 609}$, and VirD4 $4_{i 655}$ ), all mapping to the C-terminal end of the protein, did not abolish protein function.

Three mutations, two avirulent $\left(\mathrm{VirD}_{\mathrm{i} 526}\right.$ and VirD4 $\left.{ }_{\mathrm{i} 542}\right)$ and one virulent $\left(\mathrm{VirD}_{\mathrm{i} 554}\right)$, define the end of the conserved region among the VirD4 homologs. The avirulent mutations mapped to a highly charged region. Within a 34-residue segment (aa 509 to 542), 14 are charged residues (nine basic, five acidic). The equivalent region in TrwB is not charged but is required for function, because a deletion of the $\mathrm{C}$-terminal 12 or 17 residues led to severe reduction ( 3 to $4 \log$ ) in its DNA transfer activity (de Paz et al. 2010). Future studies will determine whether the polarity of the region or the secondary structure plays a role in VirD4 function.

The extreme $\mathrm{C}$ terminus of the VirD4 proteins is highly variable and is postulated to encode system-specific function. We demonstrate that this region of Agrobacterium sp. and $R$. etli VirD4 proteins contains a conserved Q-rich sequence that is essential for recognition of the VirD2-T-strand DNA as a substrate for translocation to plants (Figs. 2 and 3). This domain is not required for the recognition of a second A. tumefaciens substrate, VirE2, and that of promiscuous IncQ plasmid DNA substrate. Thus, the Q-rich domain is a specificity factor solely for the recognition of VirD2-T-strand DNA substrate. The fact that the VirD4 Q-rich domain mutants with amino acid substitutions or deletions were fully competent in VirE2 transfer suggests that a second substrate recognition site lies elsewhere in the protein (Fig. 3). The AAD domain has been proposed to function in substrate recognition (Whitaker et al. 2015).

We envision that the AAD serves as the primary substrate recognition domain. This is consistent with observations in this study and by Whitaker et al. (2016) that several VirD4 homologs (e.g., R388 TrwB, pKM101 TraJ, and Sinorhizobium medicae VirD4 [WP_011970866.1]) lack an additional Cterminal domain; that in vitro a protein containing only the AAD of A. tumefaciens VirD4 specifically bound VirD2; and that VirD4 mutants lacking the only essential domain in the Cterminal region, the Q-rich domain, are proficient in the transfer of both VirE2 and IncQ plasmid DNA. We propose that the Qrich domain modulates the primary substrate recognition domain, enabling it to recognize other substrates. The Q-rich domain probably interacts directly with the VirD2 relaxase to target VirD2-T-strand DNA to VirD4 or to stabilize interaction between VirD4 AAD and VirD2 linked to the T-strand DNA.

A low efficiency of VirD4Q633A in IncQ DNA transfer is consistent with the hypothesis that the Q-rich domain participates in protein-protein interaction. This domain is not required for IncQ plasmid DNA transfer, since VirD4 $4631-656$, a mutant with a complete deletion of the domain, as well as all mutants mapping to the Q-rich domain, except for VirD4Q633A, were proficient in pKT230 DNA transfer. The simplest explanation for the VirD4Q633A phenotype is that interaction of the mutated Q-rich domain with the DNA transfer domain is inhibitory to pKT230 transfer. The dispensable nature of the VirD4 Q-rich domain for the recognition of VirD2 in vitro reported previously (Whitaker et al. 2016) could be due to expression of the AAD in isolation. In the absence of the other domains, VirD4 AAD could acquire a conformation proficient in interaction with VirD2.

In addition, two VirD4 homologs, TraD of the E. coli $\mathrm{R}$ and $\mathrm{F}$ plasmids, contain $\mathrm{C}$-terminal protein-protein interaction domains necessary for conjugative transfer of plasmid DNA (Beranek et al. 2004; Lu and Frost 2005). The C-terminal 38 residues of $\mathrm{R}$ plasmid TraD interact with the TraM relaxosome protein. In F-TraD, the C-terminal 37 amino acids (residues 680 to 717) offer specificity for F-plasmid transfer, most likely

\begin{tabular}{|c|c|c|c|c|}
\hline $\begin{array}{c}\text { Protein } \\
\text { (Size, aa) }\end{array}$ & Source & Accession \# & Q-rich Region Sequence & $\% Q$ \\
\hline $\begin{array}{l}\text { TraD } \\
(716) \\
\end{array}$ & $\begin{array}{c}\text { F plasmid - } \\
\text { E. coli }\end{array}$ & AAA83928 & 681 QQENHPDIQQQMQ 693 & 46 \\
\hline $\begin{array}{l}\text { VirD4 } \\
\text { (639) }\end{array}$ & B. henselae & AFK10358 & 593 QEMRQDEQIILMQ 605 & 30 \\
\hline $\begin{array}{l}\text { VirD4 } \\
\text { (754) }\end{array}$ & H. pylori & EJB25423 & 738 QQGDEESEQEVLQDTQ 753 & 31 \\
\hline $\begin{array}{l}\text { VirD4 } \\
\text { (649) }\end{array}$ & $\begin{array}{l}\text { Roseibium } \\
\text { sp. }\end{array}$ & EFO28786.1 & 628 QAKADLQLEKALQVRQ 643 & 25 \\
\hline $\begin{array}{l}\text { TraD } \\
(705)\end{array}$ & V. vulnificus & BAC97747.1 & 680 QERAQQELDEAAISQDIQ 697 & 28 \\
\hline $\begin{array}{l}\text { VirD4 } \\
(382)\end{array}$ & A. faecalis & EJC62462.1 & 352 QFQPFAQVIEPFIESNQ 368 & 24 \\
\hline $\begin{array}{l}\text { TraD } \\
(556)\end{array}$ & $\begin{array}{c}\text { C. } \\
\text { amoebophila }\end{array}$ & CAF24165 & 515 QQTRYQQLITKTDIQFLSRNQ 535 & 29 \\
\hline
\end{tabular}

Fig. 5. Q-rich regions near the $\mathrm{C}$ terminus of VirD4 homologs are shown. Glutamine residues are shown in bold. 
through an interaction with the cognate relaxosome (Sastre et al. 1998). The extreme $C$ terminus of F-TraD (residues 703 to 717 ) is probably not required for F-transfer, because a 31amino acid insertion at residue 702 of F-TraD was transfer proficient (Nelson et al. 1997). The essential C-terminal F-TraD domain, therefore, is likely to lie within residues 680 to 702 (WQQENHPDIQQQMQRREEVNINV) (Fig. 5). This sequence, like that in VirD4, is rich in glutamine (6 Q/13). Several other VirD4 homologs also contain a Q-rich domain (discussed below).

Q-rich domains also are known to participate in interactions among eukaryotic proteins. Eukaryotic transcription activators, like human transcription factor Sp1, contain Q-rich domains with a hydrophobic patch (Gill et al. 1994). This domain in human Sp1 is involved in protein-protein interactions required for the assembly of the transcription factor TFIID (Gill et al. 1994; Rojo-Niersbach et al. 1999; Tanese et al. 1996). We propose a similar role for the VirD4 Q-rich domain in interaction with the VirD2 relaxase or a T4S substrate. The essential nature of multiple glutamine and bulky hydrophobic residues in VirD4 function support this hypothesis (Fig. 3). The Q-rich domain identified in this study is not unique to the Agrobacterium sp. VirD4 proteins and F TraD. Manual analysis of the C-terminal end sequences of VirD4 homologs showed that a Q-rich domain is present at or near the C-terminus of several proteins, including Bartonella henselae VirD4, Helicobacter pylori VirD4, Rosebium sp. VirD4, the marine pathogenic $\gamma$-proteobacterium Vibrio vulnificus TraD, $\beta$-proteobacterium Alcaligenes faecalis VirD4, and Chlamydiae Protochlamydia amoebophila TraD (Fig. 5). The B. henselae Q-rich domain, like that of VirD4 ${ }_{A t}$, contains large hydrophobic spacer residues. In some homologs (e.g., Wolbachia pipientis VirD4), the $\mathrm{C}$-terminal end is very acidic and is rich in aspartic acid (Whitaker et al. 2016). The role of these domains in substrate recognition remains to be determined.

\section{MATERIALS AND METHODS}

\section{Strains and plasmids.}

A. tumefaciens A136 is a derivative of strain C58 cured of its Ti-plasmid pTiC58 (Chilton et al. 1977). Agrobacterium sp. strain A348, a derivative of $A$. tumefaciens A136, harbors the octopine Ti-plasmid pTiA6NC. Strain MX358 is an A. tumefaciens A348 derivative with a transposon insertion in virE2, and A. tumefaciens LBA4404 has a deletion in the T-DNA region of the Ti-plasmid pTiAch5 (Hoekema et al. 1983; Stachel and Nester 1986). A. tumefaciens A348/pKT230 and A348/pJB61 harbor IncQ plasmid pKT230 (kanamycin resistant) and plasmid pJB61 (gentamycin resistant), respectively (Beijersbergen et al. 1992; Bohne et al.1998).

A. tumefaciens was grown overnight at $30^{\circ} \mathrm{C}$ in $\mathrm{AB}$ minimal medium with the appropriate antibiotic. For induction of virulence genes, bacteria were grown at $22^{\circ} \mathrm{C}$ in $\mathrm{AB}$ Mes $\mathrm{pH} 5.8$ minimal medium supplemented with $100 \mu \mathrm{M}$ acetosyringone as described previously (Kumar and Das 2002).

\section{Construction of A. tumefaciens virD4 deletion mutant strain A348 $\Delta$ D4.}

An unmarked deletion in the pTiA6NC virD4 was introduced by homologous recombination using $s a c \mathrm{~B}$ selection (Skerker and Shapiro 2000). DNA fragments of $1.1 \mathrm{~kb}$ (BamHI-BspHI fragment) and $1.5 \mathrm{~kb}$ (EcoRV-Bam HI fragment) from the left and right ends of virD4, respectively, were cloned into the $s a c \mathrm{~B}$ vector plasmid pNPTS138. The resultant plasmid, pPU20, was introduced into A. tumefaciens A348 by electroporation (Mersereau et al. 1990), and sucrose-resistant colonies were isolated. Several sucrose-resistant colonies were analyzed by
Southern blot hybridization and PCR amplification. A colony with the desired deletion in virD4 (codons 1 to 596 deleted) was named A. tumefaciens A348 DD4 and was used in all studies.

\section{virD4 mutagenesis.}

Random in-frame19-codon insertions within the virD4 coding region were introduced by Ez-tn5 mutagenesis (Epicentre Technologies, Madison, WI, U.S.A.). Plasmid pAD1791, a pUC119 derivative with a chimeric virDp-virD4 gene, was mutagenized in vitro with Ez-tn5 $<$ NotI/KAN-3 $>$ transposon, as per the manufacturer's instructions. Plasmids with insertions in virD 4 were identified by restriction endonuclease digestion. The site of insertion was determined by DNA sequence analysis. Eighteen unique insertions were identified from analyses of 62 mutants. The inserted transposon was then deleted by digestion of plasmid DNA with the restriction endonuclease NotI, and the plasmid was recircularized with T4 DNA ligase. This process introduced an in-frame insertion of 19 codons at the original site of the transposon insertion.

Targeted insertions of 10 in-frame codons containing a flag epitope (IDYKDDDDKL) and mutations within the Q-rich domain were introduced by deoxy-oligonucleotide directed sitespecific mutagenesis, using single-stranded pAD1791 DNA as a template (Kumar and Das 2002). The DNA sequence of oligonucleotides used for the introduction of mutations is listed in Supplementary Table S1. All plasmids were fused to the wide host range plasmid pAD1085 for analysis in A. tumefaciens. Plasmid pAD1085 was constructed by cloning a 0.7-kb DdeI fragment containing the pGEM1 polylinker region into the HindIII site of the wide host range pRK2 derivative plasmid pTJS75 (Schmidhauser and Helinski 1985). Plasmids were introduced into A. tumefaciens by electroporation.

\section{virD4 functional assays.}

The effect of a mutation in virD4 on DNA transfer was monitored by complementation assays. The ability of a mutant to complement the virD4 deletion mutant $A$. tumefaciens A348 D 4 was monitored by tumor-formation assays on Kalanchöe daigramontiana leaves (Kumar and Das 2002). Tumors were scored 3-weeks postinfection.

The effect of a mutation on T-strand DNA transfer or VirE2 transfer was monitored by in-planta complementation (Otten et al. 1984). In this assay, a wound site is coinfected with two $A$. tumefaciens-one serving as the T-DNA donor and the other serving as the VirE2 donor. In one experimental setup, the virD4 mutant served as the T-DNA donor, and A. tumefaciens LBA4404 ( $\operatorname{vir}^{+} \mathrm{T}^{-}$[Hoekema et al. 1983]) was the VirE2 donor. In the complementary set, the virD4 mutant served as the VirE2 donor, and A. tumefaciens MX358 (virE2 ${ }^{-} \mathrm{T}^{+}$[Stachel and Nester 1986]) was the T-DNA donor. Equal numbers of the two bacterial strains grown overnight in $\mathrm{AB}$ liquid medium were mixed in a microfuge tube and centrifuged to pellet the cells. Cells were resuspended at a concentration of $1 \mathrm{OD} / \mathrm{ml}$. Five microliters of the cell mixture was used to infect a leaf wound site.

\section{Conjugative transfer of an IncQ plasmid between Agrobacterium sp. strains.}

VirD4/VirB-dependent transfer of plasmid pKT230 from donor A. tumefaciens DD4/pvirD4 (or its mutant) into recipient A. tumefaciens A348 (gentamycin resistant) was monitored, essentially as described by Bohne et al. (1998). pKT230 is a kanamycin-resistant derivative of the wide host range IncQ plasmid RSF1010 (Beijersbergen et al. 1992). Donor and recipient cells were mixed (at a 1:5 ratio) and incubated for 3 days at room temperature $\left(22^{\circ} \mathrm{C}\right)$ on plates with $\mathrm{AB}$ induction medium containing $100 \mu \mathrm{M}$ acetosyringone. The cell mixture was plated on LB plates containing kanamycin/kanamycin and 
carbenicillin (to monitor donor cell concentration), gentamycin (recipient), or kanamycin and gentamycin (transconjugants). Transfer frequency is defined as the number of transconjugants recovered per donor bacterium.

\section{ACKNOWLEDGMENTS}

I thank L. Banta for providing us with A. tumefaciens used in conjugation assays, P. Uppala for construction of the Agrobacterium sp. virD4 deletion mutant strain, and H. K. Park and P. Mossey for excellent technical assistance.

\section{LITERATURE CITED}

Beijersbergen, A., Dulk-Ras, A. D., Schilperoort, R. A., and Hooykaas, P. J. 1992. Conjugative transfer by the virulence system of Agrobacterium tumefaciens. Science 256:1324-1327.

Beranek, A., Zettl, M., Lorenzoni, K., Schauer, A., Manhart, M., and Koraimann, G. 2004. Thirty-eight C-terminal amino acids of the coupling protein TraD of the F-like conjugative resistance plasmid R1 are required and sufficient to confer binding to the substrate selector protein TraM. J. Bacteriol. 186:6999-7006.

Berger, B. R., and Christie, P. J. 1994. Genetic complementation analysis of the Agrobacterium tumefaciens virB operon: virB2 through virB11 are essential virulence genes. J. Bacteriol. 176:3646-3660.

Binns, A. N., Beaupré, C. E., and Dale, E. M. 1995. Inhibition of VirBmediated transfer of diverse substrates from Agrobacterium tumefaciens by the IncQ plasmid RSF1010. J. Bacteriol. 177:4890-4899.

Bohne, J., Yim, A., and Binns, A. N. 1998. The Ti plasmid increases the efficiency of Agrobacterium tumefaciens as a recipient in virB-mediated conjugal transfer of an IncQ plasmid. Proc. Natl. Acad. Sci. U.S.A. 95: 7057-7062.

Cabezón, E., Sastre, J. I., and de la Cruz, F. 1997. Genetic evidence of a coupling role for the TraG protein family in bacterial conjugation. Mol. Gen. Genet. 254:400-406.

Cascales, E., Atmakuri, K., Liu, Z., Binns, A. N., and Christie, P. J. 2005. Agrobacterium tumefaciens oncogenic suppressors inhibit T-DNA and VirE2 protein substrate binding to the VirD4 coupling protein. Mol. Microbiol. 58:565-579.

Cascales, E., Atmakuri, K., Sarkar, M. K., and Christie, P. J. 2013. DNA substrate-induced activation of the Agrobacterium VirB/VirD4 type IV secretion system. J. Bacteriol. 195:2691-2704.

Chilton, M.-D., Drummond, M. H., Merio, D. J., Sciaky, D., Montoya, A. L., Gordon, M. P., and Nester, E. W. 1977. Stable incorporation of plasmid DNA into higher plant cells: The molecular basis of crown gall tumorigenesis. Cell 11:263-271.

Christie, P. J. 2001. Type IV secretion: Intercellular transfer of macromolecules by systems ancestrally related to conjugation machines. Mol. Microbiol. 40:294-305.

Christie, P. J., Whitaker, N., and González-Rivera, C. 2014. Mechanism and structure of the bacterial type IV secretion systems. Biochim. Biophys. Acta 1843:1578-1591.

Das, A. 1988. Agrobacterium tumefaciens virE operon encodes a singlestranded DNA-binding protein. Proc. Natl. Acad. Sci. U.S.A. 85:2909-2913.

Das, A., and Xie, Y.-H. 1998. Construction of transposon Tn3phoA: Its application in defining the membrane topology of the Agrobacterium tumefaciens DNA transfer proteins. Mol. Microbiol. 27:405-414.

de Paz, H. D., Larrea, D., Zunzunegui, S., Dehio, C., de la Cruz, F., and Llosa, M. 2010. Functional dissection of the conjugative coupling protein TrwB. J. Bacteriol. 192:2655-2669.

Escoll, P., Rolando, M., Gomez-Valero, L., and Buchrieser, C. 2013. From amoeba to macrophages: Exploring the molecular mechanisms of Legionella pneumophila infection in both hosts. Curr. Top. Microbiol. Immunol. 376:1-34.

Fronzes, R., Schäfer, E., Wang, L., Saibil, H. R., Orlova, E. V., and Waksman, G. 2009. Structure of a type IV secretion system core complex. Science 323:266-268.

Gelvin, S. B. 2012. Traversing the cell: Agrobacterium T-DNA's journey to the host genome. Front. Plant Sci. 3:52.

Gill, G., Pascal, E., Tseng, Z. H., and Tjian, R. 1994. A glutamine-rich hydrophobic patch in transcription factor Sp1 contacts the dTAFII110 component of the Drosophila TFIID complex and mediates transcriptional activation. Proc. Natl. Acad. Sci. U.S.A. 91:192-196.
Gomis-Rüth, F. X., Moncalían, G., de la Cruz, F., and Coll, M. 2002. Conjugative plasmid protein TrwB, an integral membrane type IV secretion system coupling protein. Detailed structural features and mapping of the active site cleft. J. Biol. Chem. 277:75567566.

Gomis-Rüth, F. X., Moncalián, G., Pérez-Luque, R., González, A., Cabezón, E., de la Cruz, F., and Coll, M. 2001. The bacterial conjugation protein TrwB resembles ring helicases and F1-ATPase. Nature 409:637-641.

Haft, R. J. F., Gachelet, E. G., Nguyen, T., Toussaint, L., Chivian, D., and Traxler, B. 2007. In vivo oligomerization of the F conjugative coupling protein TraD. J. Bacteriol. 189:6626-6634.

Hoekema, A., Hirsch, P. R., Hooykaas, P. J. J., and Schilperoort, R. A. 1983. A binary plant vector strategy based on separation of vir- and T-region of the Agrobacterium tumefaciens Ti-plasmid. Nature 303:179-180.

Kumar, R. B., and Das, A. 2002. Polar location and functional domains of the Agrobacterium tumefaciens DNA transfer protein VirD4. Mol Microbiol. 43:1523-1532.

Lu, J., and Frost, L. S. 2005. Mutations in the C-terminal region of TraM provide evidence for in vivo TraM-TraD interactions during F-plasmid conjugation. J. Bacteriol. 187:4767-4773.

Mersereau, M., Pazour, G. J., and Das, A. 1990. Efficient transformation of Agrobacterium tumefaciens by electroporation. Gene 90:149-151.

Nelson, B. D., Manoil, C., and Traxler, B. 1997. Insertion mutagenesis of the lac repressor and its implications for structure-function analysis. J. Bacteriol. 179:3721-3728.

Otten, L., DeGreve, H., Leemans, J., Hain, R., Hooykaas, P., and Schell, J. 1984. Restoration of virulence of vir region mutants of Agrobacterium tumefaciens strain B6S3 by coinfection with normal and mutant Agrobacterium strains. Mol. Gen. Genet. 195:159-163.

Rojo-Niersbach, E., Furukawa, T., and Tanese, N. 1999. Genetic dissection of $\mathrm{hTAF}_{\mathrm{II}} 130$ defines a hydrophobic surface required for interaction with glutamine-rich activators. J. Biol. Chem. 274:33778-33784.

Sastre, J. I., Cabezón, E., and de la Cruz, F. 1998. The carboxyl terminus of protein TraD adds specificity and efficiency to F-plasmid conjugative transfer. J. Bacteriol. 180:6039-6042.

Schmidhauser, T. J., and Helinski, D. R. 1985. Regions of broad-host-range plasmid RK2 involved in replication and stable maintenance in nine species of gram-negative bacteria. J. Bacteriol. 164:446-455.

Simone, M., McCullen, C. A., Stahl, L. E., and Binns, A. N. 2001. The carboxy-terminus of VirE2 from Agrobacterium tumefaciens is required for its transport to host cells by the virB-encoded type IV transport system. Mol. Microbiol. 41:1283-1293.

Skerker, J. M., and Shapiro, L. 2000. Identification and cell cycle control of a novel pilus system in Caulobacter crescentus. EMBO J. 19: 3223-3234

Stachel, S. E., and Nester, E. W. 1986. The genetic and transcriptional organization of the vir region of the A6 Ti plasmid of Agrobacterium tumefaciens. EMBO J. 5:1445-1454.

Tanese, N., Saluja, D., Vassallo, M. F., Chen, J.-L., and Admon, A. 1996. Molecular cloning and analysis of two subunits of the human TFIID complex: hTAFII130 and hTAFII100. Proc. Natl. Acad. Sci. U.S.A. 93: 13611-13616.

Tato, I., Matilla, I., Arechaga, I., Zunzunegui, S., de la Cruz, F., and Cabezon, E. 2007. The ATPase activity of the DNA transporter TrwB is modulated by protein TrwA: Implications for a common assembly mechanism of DNA translocating motors. J. Biol. Chem. 282:25569-25576.

Vergunst, A. C., Schrammeijer, B., den Dulk-Ras, A., de Vlaam, C. M., Regensburg-Tuïnk, T. J., and Hooykaas, P. J. 2000. VirB/D4-dependent protein translocation from Agrobacterium into plant cells. Science 290 979-982.

Waters, C. M., and Dunny, G. M. 2001. Analysis of functional domains of the Enterococcus faecalis pheromone-induced surface protein aggregation substance. J. Bacteriol. 183:5659-5667.

Whitaker, N., Berry, T. M., Rosenthal, N., Gordon, J. E., Gonzalez-Rivera, C., Sheehan, K. B., Truchan, H. K., VieBrock, L., Newton, I. L. G., Carlyon, J. A., and Christie, P. J. 2016. Chimeric coupling proteins mediate transfer of heterologous type IV effectors through the Escherichia coli pKM101-encoded conjugation machine. J. Bacteriol. 198:2701-2718.

Whitaker, N., Chen, Y., Jakubowski, S. J., Sarkar, M. K., Li, F., and Christie, P. J. 2015. The all-alpha domains of coupling proteins from the Agrobacterium tumefaciens VirB/VirD4 and Enterococcus faecalis pCF10-encoded type IV secretion systems confer specificity to binding of cognate DNA substrates. J. Bacteriol. 197:2335-2349. 